\title{
A Low-Complexity Mobile Watermarking Scheme Resisting Scale Distortions
}

\author{
Dai-Kyung Hyun, Heung-Kyu Lee* \\ Department of Computer Science, Korea Advanced Institute of Science and Technology, Daejeon, Korea \\ Email: $\underline{\text { hklee@mmc.kaist.ac.kr }}$
}

Received November 2013

\begin{abstract}
In mobile environment, a low-complexity is the significant feature because the mobile device has very limited resources due to power consumption. In this paper, we propose a low-complexity watermarking scheme for mobile device. We apply the minimum average correlation energy Mellin radial harmonic (MACE-MRH) correlation filter to watermark detection. By the scale tolerance property of MACE-MRH correlation filter, the proposed watermark detector can be robust to scaling attacks. Empirical evidence from a large database of test images indicates outperforming performance of the proposed method.
\end{abstract}

\section{Keywords}

Low-Complexity; Mobile Watermarking; MACE-MRH Correlation Filter

\section{Introduction}

With the development of mobile devices, the copyright protection on mobile devices has become more important. One of the best methods for copyright protection is digital watermarking. Digital watermarking is a solution to the copy protection by inserting a pattern of bits, which identifies the digital content's copyright information, into digital content [1,2]. Digital watermarking offers persistent digital identify carried directly within content. Thus, in the mobile environment, it can be easily applied.

In real-world watermarking scenario, the watermark should be robust to scaling attacks because digital contents can be easily scaled. Many watermarking methods resilient to geometric distortion such as scaling were reported. Pereira and Pun proposed an approach to embed a digital watermark into an image using the Fourier transform [3]. This approach efficiently recovered the template in an image which has undergone a general affine transformation. In [4], Fourier-Mellin transform based approach was proposed. Their method utilizes geometric invariant property of Fourier-Mellin transform. The method was unaffected by any combination of rotation, scale and translation transformations. Alghoniemy and Tewfik presented a watermarking scheme based on its moments. The watermark was composed of the mean of several functions of the second and third order scaling invariant moments. Their method was to be robust to geometric manipulations [5].

In mobile environment, one of the significant features is low complexity because the mobile device has very

\footnotetext{
${ }^{*}$ Corresponding author.
} 
limited resources due to power consumption. Thus, the watermarking method should have a low-complexity. However, the previous works require complicated calculations such as wavelet transform and Fourier-Mellin transform in the process of watermark embedding and extracting. Therefore, we propose a low-complexity watermarking method to be robust scaling attacks.

To be robust to scaling distortion, we adopt the minimum average correlation energy Mellin radial harmonic (MACE-MRH) correlation filter to watermark detection. The MACE-MRH correlation filter allows specifying the scaling response of the correlation filter. Thus, this filter has the scaling tolerance property. It is required a complicated computation to generate the MACE-MRH correlation filter. However, it can be designed off-line and needs only one generation of the filter during the watermark detection. Once the filter is generated, it only requires a calculation of cross correlation. Therefore, the proposed method has very low complexity.

The rest of this paper is organized as follows. In Section 2, we describe the MACE-MRH correlation filter. Our watermarking scheme is presented in Section 3. Experimental results are shown in Section 4 and Section 5 which concludes the paper.

\section{MACE-MRH Correlation Filter [6]}

In this section, we describe MACE-MRH correlation filter design theory. The MACE-MRH correlation filter is based on the Mellin radial harmonic (MRH) transform, given by the following pair of equations:

$$
\begin{aligned}
& M(\rho, \phi)=\sum_{m=-\infty}^{\infty} M_{m}(\phi) \rho^{j 2 \pi m-1} \\
& \text { where } M_{m}(\phi)=L^{-1} \int_{d}^{R} M(\rho, \phi) \rho^{-j 2 \pi m-1} \rho d \rho
\end{aligned}
$$

where $M(\rho, \varphi)$ is the FT of a 2-D pattern $\mathrm{m}(\mathrm{x}, \mathrm{y})$ in polar coordinates and $M_{m}(\varphi)$ is the m-th MRH function of $M(\rho, \varphi)$.

The scale invariance property of the MRH transform is explained as follows. If $\mathrm{g}_{\beta}(\mathrm{x}, \mathrm{y})$ is a scaled version of 2-D pattern $\mathrm{g}(\mathrm{x}, \mathrm{y})$, i.e., $\mathrm{g}_{\beta}(\mathrm{x}, \mathrm{y})=\mathrm{g}(\beta \mathrm{x}, \beta \mathrm{y})$, then the $\mathrm{m}$-th MRH function of the 2-D polar FT of $\mathrm{g}_{\beta}(\mathrm{x}, \mathrm{y})$ is given by

$$
G_{\beta m}(\phi)=L^{-1} \beta^{-j 2 \pi m-1} G_{m}(\phi)
$$

where $G_{m}(\varphi)$ is the m-th MRH function of the 2-D polar FT of $\mathrm{g}(\mathrm{x}, \mathrm{y})$. Thus, the scaling operation only affects the MRH transform coefficient functions by a phase factor $\beta^{-j 2 \pi m-1}$.

If the 2-D polar FT of the reference pattern and the correlation filter in polar coordinates are represented as $F$ and $H$, respectively, we obtain the following expression for the correlation value c between $F(\rho, \varphi)$ and $H(\rho, \varphi)$.

$$
c=\int_{0}^{2 \pi} \int_{d}^{R} F(\rho, \phi) H^{*}(\rho, \phi) \rho d \rho d \phi .
$$

If $\mathrm{L}=\ln (\mathrm{R} / \mathrm{d})$ in Equation (1) is an integer, the MRH components are orthogonal. Equation (3) can be decomposed in terms of MRH decompositions in Equation (1).

$$
c=L \sum_{m=-\infty}^{\infty} C_{m}, C_{m}=\int_{0}^{2 \pi} F_{m}(\phi) H_{m}^{*}(\phi) d \phi .
$$

Supposing a scaled pattern $\mathrm{f}_{\beta}(\mathrm{x}, \mathrm{y})=\mathrm{f}(\beta \mathrm{x}, \beta \mathrm{y})$, we can rewrite Equation (4) by the scale invariance property of Equation (2) as a function of the scale factor $\beta$ :

$$
c(\beta)=\beta^{-1} \sum_{m=-\infty}^{\infty} e^{-j 2 \pi m \ln \beta} C_{m} .
$$

Equation (5) can be simplified by a invertible logarithmic transformation $\Lambda$ so that we yield $c(\beta)$ in Equation (6)

$$
\hat{c}(\beta)=\wedge\{c(\beta)\}=e^{\frac{L \beta}{2 \pi}} \cdot c\left(e^{\frac{L \beta}{2 \pi}}\right)=\sum_{m=-\infty}^{\infty} C_{m} e^{-j(L m) \beta} .
$$

The relationship between the transformed scale response $c(\beta)$ and the coefficients $C_{m}$ is exactly the same as 
$\mathrm{A}(\mathrm{w})$ and $a_{k}$ of Equation (6), which represent the frequency response of the finite impulse response (FIR) filter and the k-th coefficient of the impulse response, respectively.

$$
A(\omega)=\sum_{k=-M}^{M} a_{k} e^{-j k \omega}
$$

The similarity of Equation (6) and Equation (7) can make the problem of calculating MRH weighting values $\mathrm{C}_{\mathrm{m}}$ as that of designing the FIR filter of $A(\omega)$. Thus, by employing available a FIR filter design algorithm [7], we can calculate the weighting values $C_{m}$ of $c(\beta)$.

Once the $C_{m}$ are determined, they can be used as constraints for determining MRH function $H_{m}(\varphi)$ of Equation (5). However, for each $\mathrm{m}$, there are many $H_{m}(\varphi)$ candidates that satisfy the constraints. Thus, more constraints are needed to find a unique solution. Therefore, we select $H_{m}(\varphi)$ that minimize the average correlation energy (ACE) while satisfying the relationship in Equation (5), thereby maximizing the sharpness of output peak while achieving a specified scale response $c(\beta)$. The ACE is equal to the average energy of the correlation filter as described in Equation (8).

$$
A C E=L \sum_{m=-\infty}^{\infty}\left[\int_{0}^{2 \pi}\left|H_{m}(\phi)\right|^{2} P_{F}(\phi) d \phi\right]
$$

where $P_{F}(\varphi)$ is the average power spectrum of the reference pattern obtained by computing the average of $F(\rho$, $\varphi)^{2}$ along the radial axis.The MACE-MRH correlation filter is designed to minimize the ACE in Equation (8) while satisfying the relationship in Equation (5). Thus, the filter $\mathrm{H}(\mathrm{u}, \mathrm{v})$ can be obtained by solving the following minimization problem.

$$
\begin{aligned}
& \text { Find } H_{m}(\phi) \text { to minimize } \\
& \qquad \int_{0}^{2 \pi}\left|H_{m}(\phi)\right|^{2} P_{F}(\phi) d \phi
\end{aligned}
$$

subject to

$$
\int_{0}^{2 \pi} F_{m}(\phi) H_{m}^{*}(\phi) d \phi=C_{m}
$$

Using the method of Lagrange multipliers, the solution to the minimization problem of Equation (9) is

$$
H_{m}(\phi)=\lambda_{m}^{*} \frac{F_{m}(\phi)}{P_{F}(\phi)+\alpha}, \lambda_{m}=\frac{F_{m}(\phi)}{\int_{0}^{2 \pi} \frac{\left|F_{m}(\phi)\right|^{2}}{P_{F}(\phi)+\alpha} d \phi}
$$

where $\alpha$ is a small additive constant. Finally, the correlation filter $\mathrm{H}(\mathrm{u}, \mathrm{v})$ is found by applying the inverse MRH transforms of $H_{m}(\varphi)$ and applying a polar-to-cartesian coordinate transform.

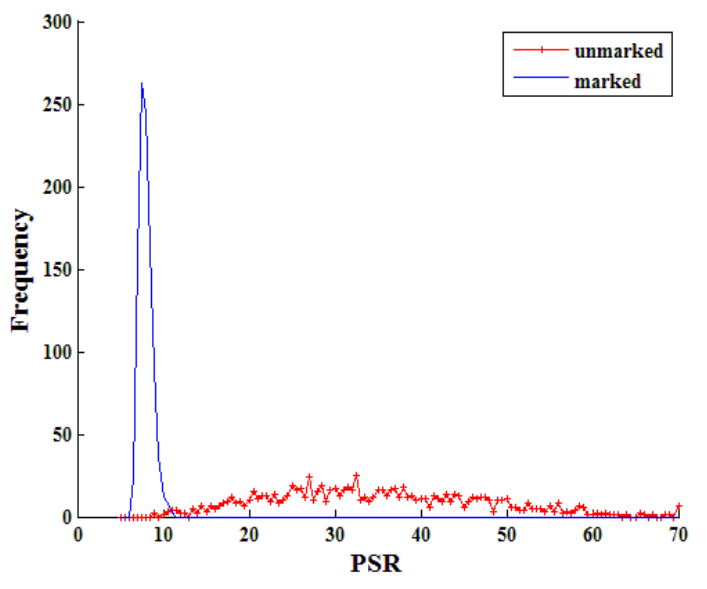

(a)

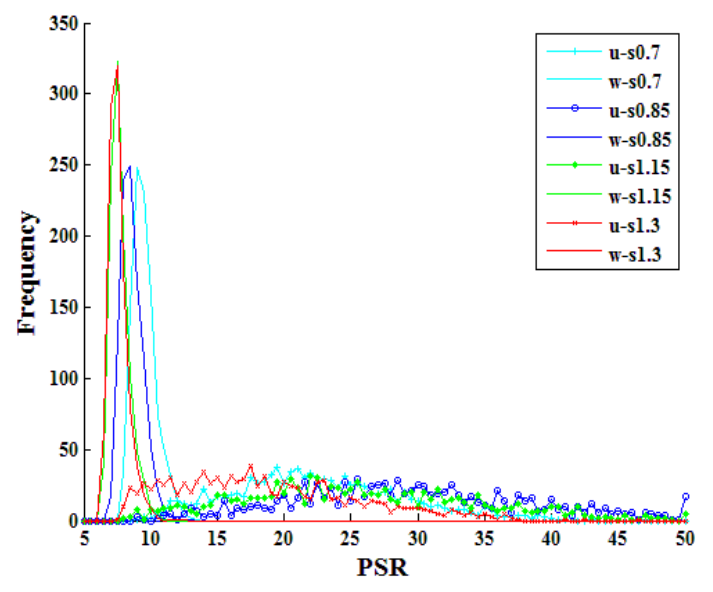

(b)

Figure 1. Empirical detector performance: (a) Before scaling; (b) After scaling. 


\section{Proposed Method}

The previous section described how a MACE-MRH correlation filter was designed. In this section, it is explained how the MACE-MRH correlation filter is applied to the proposed watermark detection.

\subsection{Watermarking Embedding}

We propose a simple additive watermarking method for low-complexity. First, a simple perceptual marking matrix $\mathrm{M}$ is calculated as below:

$$
M=|F * I|, F=\left[\begin{array}{ccc}
-1 & -1 & -1 \\
-1 & 8 & -1 \\
-1 & -1 & -1
\end{array}\right]
$$

where $I$ is a sample image.

After that, the watermarked image $Y$ is computed as follows:

$$
Y=X+F W
$$

where $W$ is a white watermark.

The embedding process has very low-complexity. Therefore, it can be suitably applied on the mobile environment.

\subsection{Watermark Detection}

To be robust to scaling attacks, we adopt the MACE-MRH correlation filter designed from watermark pattern $W$ of Equation (12). We design MACE-MRH correlation filter from watermark pattern be determining the scale response $c(\beta)$ as follows:

$$
H=f_{0}(W, c(\beta)), c(\beta)=\left\{\begin{array}{l}
1, \text { if }|\beta| \leq \beta_{t} \\
0, \text { otherwise }
\end{array}\right.
$$

where $\mathrm{f}_{0}$ is the filter design function of Equation (10). This procedure would be required a complicated computation. However, it needs only one generation of the filter during the watermark detection.

After generating MACE-MRH correlation filter, the proposed method requires only a computation of crosscorrelation as follow:

$$
\begin{aligned}
& c c(x, y)=\sum_{m=0}^{M} \sum_{n=0}^{N} \widetilde{H}(m, n) \cdot \tilde{W}(m+x, n+y) \\
& \text { where } \tilde{H}=f_{\text {filter }}(H), \widetilde{W}=I-f_{\text {filter }}(I)
\end{aligned}
$$

From the correlation result, the proposed method determined whether watermark was embedded or not as follow:

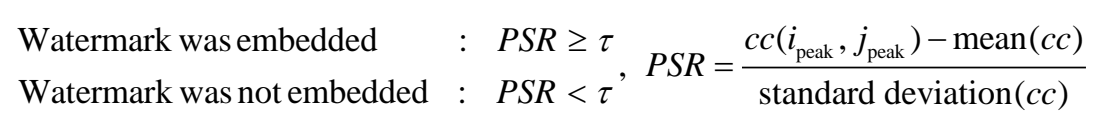

where $\left(i_{\text {peak }}, j_{\text {peak }}\right)$ are the peak position in $c c(\mathrm{x}, \mathrm{y})$ and $\tau$ is threshold.

\section{Experimental Results}

The experiment was performed with 1000 watermarked images with $\beta=0.5$. To demonstrate the performance about scaling attacks, the images were resized in increments of 0.15 from 0.7 to 1.3 . With the watermarked images, we measured the resulting correlation performances in term of peak to side robe ratio (PSR) of Equation (15). Figure 1 shows the PSR histogram of un-scaled image and scaled image. It shows that the proposed method is robust to scaling attacks.

We also calculate the average detection rate by determined threshold $\tau$ of Equation (15) to false positive rate $(\mathrm{FPR})=10^{-3}$. Table 1 shows the average detection rate on FPR $=10^{-3}$. The experimental result indicates that the proposed method maintains high performance with scaled images. 
Table 1. Detection rate (\%) at FPR $=10^{-3}$.

\begin{tabular}{cc}
\hline Scaling factor & Detection rate (\%) \\
\hline 0.7 & 94.6 \\
0.85 & 97.9 \\
1 & 98.2 \\
1.15 & 93.6 \\
1.3 & 92.7 \\
\hline
\end{tabular}

\section{Conclusion}

In this paper, we introduced a low-complexity mobile watermarking scheme. By applying MACE-MRH correlation filter to a simple additive watermarking, our method has very low-complexity and is robust to scaling attacks. The experimental results show that the proposed method can be detected watermark from scaled images.

\section{Acknowledgements}

This work was partially supported by Defense Acquisition Program Administration and Agency for Defense Development under the contract (UD060048AD).

\section{References}

[1] Cox, I.J. and Miller, M.L. (2001) Electronic Watermarking: The First 50 Years. Proceedings of IEEE International Workshop on Multimedia Signal Processing, 225-230.

[2] Hartung, F. and Kutter, M. (1999) Multimedia Watermarking Technique. Proceedings of IEEE, 87, 1079-1107. http://dx.doi.org/10.1109/5.771066

[3] Pereira, S. and Pun, T. (2000) Robust Template Matching for Affine Resistant Image Watermarks. IEEE Transactions on Image Processing, 9, 1123-1129. http://dx.doi.org/10.1109/83.846253

[4] O’Ruanaidh, J.J.K. and Pun, T. (1998) Rotation, Scale, and Translation Invariant Spreadspectrum Digital Image Watermarking. Signal Processing, 66, 303-317. http://dx.doi.org/10.1016/S0165-1684(98)00012-7

[5] Alghoniemy, M. and Tewfik, A.H. (2000) Image watermarking by moment invariant. IEEE International Conference on Image Processing, 73-76.

[6] Kerekes, J.R. and Kumar, B. (2006) Correlation Filters with Controlled Scale Response. IEEE Transactions on Image Processing, 15, 1794-1802. http://dx.doi.org/10.1109/TIP.2006.873468

[7] Oppenheim, A.V. and Schafer, R.W. (1975) Discrete-Time Signal Processing. Prentice-Hall, Englewood Cliffs. 